\title{
Visual analysis of sensor logs in smart spaces: Activities vs. situations
}

\author{
Francesco Leotta, Massimo Mecella, Daniele Sora \\ Sapienza Università di Roma \\ Email: $\{$ leotta, mecella, sora\} @diag.uniromal.it
}

\begin{abstract}
Models of human habits in smart spaces can be expressed by using a multitude of representations whose readability influences the possibility of being validated by human experts. Our research is focused on developing a visual analysis pipeline (service) that allows, starting from the sensor $\log$ of a smart space, to graphically visualize human habits. The basic assumption is to apply techniques borrowed from the area of business process automation and mining on a version of the sensor log preprocessed in order to translate raw sensor measurements into human actions. The proposed pipeline is employed to automatically extract models to be reused for ambient intelligence. In this paper, we present an user evaluation aimed at demonstrating the effectiveness of the approach, by comparing it wrt. a relevant state-of-the-art visual tool, namely SITUVIS.
\end{abstract}

\section{Introduction}

The aim of a smart space is providing people with automatic or semi-automatic services realizing the concept of ambient intelligence (AmI). The input for these intelligent services is represented by a sensor $\log$, which is a sequence of measurement values acquired from sensors deployed across the monitored space. According to the number and type of installed sensors, and on the number of users acting in the environment, the amount of data produced may vary grandly in terms of size and rate. Additionally, the increasing availability of low-cost sensing technologies, even on wearable devices and smartphones, makes it likely to imagine a near future where a space (e.g., an house) and its inhabitants produce huge volumes and rates of data, according to the vision of the Internet-of-Things (IoT). Indeed, according to a classification proposed by UNECE (United Nations Economic Commission for Europe $\sqrt{1}$ there are three main types of data sources that can be viewed as big data: human sourced (e.g., blog comments), process mediated (e.g., banking records), and machine generated. Sensor measurements fall into this latter category.

The work of Daniele Sora has been partly supported by the Lazio regional project SAPERI \& Co - FILAS-RU-2014-1113, the work of Francesco Leotta has been partly supported by the Lazio regional project Sapientia - FILAS-RU-2014-1186, all the authors have been also partly supported by the Italian projects NEPTIS - PON03PE_00214_3, SM\&ST CTN01_00034_23154 and RoMA - SCN_00064.

1. cf. UNECE. Classification of types of big data. https://statswiki.unece. org/display/bigdata/Classification+of+Types+of+Big+Data Accessed January 2018
Many approaches have been proposed in the literature to represent how sensor measurements are related one to each other through models. Models can be either manually defined (specification-based methods) or obtained through machine learning techniques (learning-based methods). In the first case, models are usually based on logic formalisms, relatively easy to read and validate (once the formalism is known to the reader), but their creation requires a major cost in terms of expert time and effort. In the latter case, the model is automatically learned from a training set (whose labeling cost may vary according to the proposed solution) but employed formalisms are usually taken from statistics, making them less immediate to understand.

The cost (effort and time of experts) required by specification-based methods can rapidly become unsustainable if sensor measurements are directly used as atomic terms (i.e, basic modeling elements) of the models. As a consequence, such models usually employ high-level actions and events as basic terms. Learning-based methods usually directly refer to sensor measurements, thus loosing the focus on human actions and making even more difficult to visually inspect and validate produced models. On the other hand, taking as input raw sensor measurements usually makes learning-based methods easier to apply in a practical context; whereas, in the vast majority of cases, specificationbased methods do not face (and solve) the problem of translating sensor measurements into actions.

Another important classification of models is based on those ones taking into account the order in which events are detected and those that instead focus on the overall situation of the environment. An example is SITUVIS [1], one of the few approaches adopting visual analytics for analyzing smart spaces.

Initial approaches to the development of context-aware systems able to recognize situations were based on predicate logic, cf. [2], [3]; also ontologies have been employed in identifying situations [4]-[6]. [7] introduces the concept of Active DataBase (ADB) composed by Event-ConditionAction (ECA) rules: an ECA rule basically has the form "ON event IF condition THEN action", where conditions can take into account time. The APUBS [8] system extends this approach by mining ECA rules instead of relying on a specification-based approach.

As argued in [9], applying methods, originally taken from the area of business process management (BPM, cf. [10]) and more specifically of process mining [11], to human habits may represent a compromise between 
specification-based and learning-based methods, provided that the gap between raw sensor measurements and human actions can be filled in by performing a log preprocessing step. The first attempts were the employment of workflow specifications to anticipate user actions. A workflow is composed by a set of tasks related by qualitative and/or quantitative time relationships. [12] presents a survey of techniques for temporal calculus (i.e., Allen's Temporal Logic and Point Algebra) and spatial calculus aiming at decision making. The SPUBS system [13], [14] automatically retrieve these workflows from sensor data. [15], [16] propose both a supervised and an unsupervised technique to fill the gap between sensor raw measurements and process tasks to then apply an inductive miner and obtain a Petri net (representing the workflow).

A typical approach to deal with unstructured processes is fuzzy mining [17]; it borrows concepts from maps and cartography and applies them to zoom in and out on a process model highlighting the importance of certain tasks and connection between tasks, just like they were points and paths on a map. Our previous work [18], [19] proposes a method to exploit fuzzy mining to automatically extract models of human activities.

So far, a few studies have considered an user evaluation among the different approaches; in this paper, we present a comparative user evaluation between the fuzzy modeling from our previous work [18], [19] and the situation-based method described in [1]. The following of this paper is organized as it follows: Section 2 introduces the backgroud needed to present the comparative evaluation, which is then presented in Section 3. Finally Section 4 presents the conclusions that can be drawn from the evaluation.

\section{Background}

A smart space produces, at runtime, a sensor log containing raw measurements from available sensors. Measurements can be produced by a sensor on a periodic base (e.g., temperature) or whenever a particular event is detected (e.g., a door opening). Many solutions, especially those based on pattern analysis, expect as input an event log instead. Events are usually obtained by filtering and aggregating measurements from the sensor log; thus its granularity, in general, is coarser than the one observed in the sensor log and can potentially lead to loss of information.

Authors use, sometimes as synonyms, a variety of terms to refer to the state of the environment and the tasks humans perform in it. In this paper, we will use the following terminology (we extend here the one proposed in [20]):

Action: atomic interaction with the environment or a part of it (e.g., a device). Recognizing actions can be more or less difficult depending on the sensors installed.

Activity: a sequence of actions (one in the extreme case) or sensor measurements/events with a final goal. In some cases an action can be an activity itself (e.g., ironing). Activities can be collaborative, including actions by multiple users, and can interleave one each other. The granularity of considered activities cannot be precisely specified. According to the given approach, tidying up a room can be an activity whereas other approaches may generically consider tidying up the entire house as an activity. In any case, some approaches may hierarchically define activities, where an activity is a combination of sub-activities.

Context: the state of the environment including both sensors, actuators and the human inhabitants. The state of a human inhabitant includes the action/s and the activity/ies he/she is performing.

Situation: a high level interpretation of the context, e.g., a meeting is happening. As such, it can encompass the state of multiple devices and human inhabitants, taking into account the activities each of them is performing.

Sensor and event logs can be used to automatically discover models of human habits. These models can take many different forms, but the fundamental idea is to describe constraints between events onset and their relationships with the state of the environment. There are many different ways these models can be used, including anomaly detection, automatic adaptation, recommender systems. The granularity of the obtained model strongly influences the possible applications of the model itself for smart space automation.

In turn, what can be mined is heavily constrained by the type of sensors that are available in the environment. As an example, with only presence infrared sensors, whatever technique can only mine as habit the "laying in the bed", but cannot distinguish specific actions performed in the bed (e.g., sleeping, reading a book, any other activity); conversely, if a sensor on the bedside lamp and a sleep tracker are available, then actions can be better identified.

In BPM, a business process is a collection of related events, activities, and decisions that involve a number of actors and resources and that collectively lead to an outcome that is of value for an organization or a user. The process logic is explicitly described in terms of a process schema (i.e., the model), and a specific execution of a process is named process instance, or also case. The progress of a process instance produces a trace of execution, which may be stored in an event log and can be used for process mining [11], e.g., discovering a process model from the event log or checking the compliance of the log with the model.

\subsection{Fuzzy mining}

Fuzzy mining consists of an offline phase and an online phase. During the offline phase, a set of metrics is computed. A metric can be either unary or binary. Unary metrics are computed on single tasks (i.e., event classes), whereas binary metrics are computed on the precedence relation between two tasks. Two fundamental types of metrics are computed: (i) significance and (ii) correlation.

Significance metrics, which can be both unary and binary, measure the absolute importance of an event or a precedence relation. As such, it specifies the level of interest we have in single event classes, or in specific couples of event classes. Correlation metrics, on the other hand, 
are only binary metrics, thus they are only computed for precedence relations over events and measure how closely related two event classes are. It is worth to note how an event $e_{1}$ of class $E_{1}$ precedes another event $e_{2}$ of class $E_{2} \neq E_{1}$ even when they do not immediately follow each other in the $\log$. The number of events between $e_{1}$ and $e_{2}$ is called distance. In order to take into account this distance, attenuation functions are employed. Examples for measuring correlation include determining the overlap of attributes associated to two events following one another, or comparing the similarity of their event names. More closely correlated events are assumed to share a large amount of their attribute values, or have their similarity expressed in their recorded names (e.g., "staying in the bed" and "laying in the bed").

All significance and correlation metrics for a single event class or a single precedence relations are combined into aggregated measures. Thus, if no filtering is applied, the output of fuzzy mining is a weighted directed graph where nodes are tasks and an arc from task $T_{1}$ to $T_{2}$ with weight $w$ states that $T_{2}$ follows $T_{1}$ (respectively $T_{1}$ precedes $T_{2}$ ) $w$ times. The weight of a node (i.e., an event class) is its aggregated significance, whereas two weights are assigned to each edge: an aggregated significance and an aggregated correlation metric.

During the second phase, i.e., the online one, analytics can be performed by a human user by tuning the parameters of a visualizer. By tuning the parameters of fuzzy mining, it is possible to zoom in and out the model by highlighting paths that are significant with respect to the zoom level. This allows to focus only on the important aspects of the model. Implementations of the fuzzy miner are available as either open source (e.g., as plugin of the ProM framework) or commercial (e.g., Disco).

At the best of our knowledge, no formal validation of the readability, from a final user point of view, of fuzzy mining have been conducted. Despite this, fuzzy mining (and Disco Fuzzy miner in particular) are used with success in a variety of industrial and civil application fields as witnessed during the process mining camp initiative held by Fluxicon (the software house building Disco) every year (cfr. https://fluxicon.com/camp/2017/ for the last edition).

\subsection{Situvis}

The importance of providing domain experts with a tool allowing to graphically inspect models of smart spaces is remarked in [1], where SITUVIS is presented. The SITUVIS tool contains two separate interfaces, named according to the principal visualization methods used in each: the Timeseries (TS) view and the Parallel Coordinates (PC) view. The TS view consists of a time-series representation of the data and a panel for selection of annotated traces and classified traces. The PC view gives users a global view of trends in the data while allowing direct interaction to filter the data set as desired. A set of parallel vertical axes are drawn, which correspond to attributes of the readings in the system. In this case, the readings are records of context data at a certain time, with each axis representing a sensor in the system. Then, a set of n-dimensional tuples are drawn as a set of polylines, i.e., lines drawn starting at the leftmost axis and continuing rightwards to the next adjacent and so on, intersecting each axis at the point that represents the value that the context has in that trace. For example if, in a given situation, a user's computer activity level is "idle", and the location is "canteen", and these two axes are adjacent, then a line will be drawn between those two points. Each axis has a "no value" point, which represents that this dimension is missing from any trace whose polyline passes through it. Polylines are not drawn for times when no traces were recorded. Each trace is plotted on the axes and the result is a view of all the traces, significant and insignificant, that occurred in the system over a period of time. Discrete and quantitative axes can be presented in the same view [1]. As all the polylines are being drawn within the same area, the technique scales well to large data sets with arbitrary numbers of attributes, presenting a compact view of the entire data set. Axes can be appended or removed from the visualization as required by the dimensions of the data.

As Parallel Coordinates have a tendency to become crowded as the size of the data set grows larger, techniques have been designed to cluster or elide sub-sets of the data to allow the dominant patterns to be seen. Authors of SITUVIS use a technique to group case lines that are assigned to a certain situation, color-coding these as a group. Different situations can be color-coded so that the interplay of the context traces that correspond to them can be easily seen.

\section{The comparative user evaluation}

In our previous work, we have proposed fuzzy processing modeling as a readable and understandable representation of habits mined in a smart environment. We have proposed a service, named VPM (Visual Process Maps), focused on providing the representation of a complete habit: by exploiting fuzzy mining, obtained models can show some features of the habits, as for instance the most representative action and which are the main correlations between two actions in the single activity context or for the complete daily habit. The interested reader can refer to [19] for the details of the pipeline composing the service .

There are other tools, as for instance SITUVIS [1], focused on the graphical representation of activities. In particular, SITUVIS is designed to provide a graphical representation of situations; in [1] (similarly to definition given in Section 2), situations are defined as "high-level abstractions that serve as a suitable model with which to develop contextaware systems". By employing parallel coordinates, the tool can give an immediate view of different system statuses detected during the recurrence of a given situation. Additionally, the variability of feature values between different situation instances easily allows to understand which are the variable ranges representative of each situation.

As a consequence, even if targeted to the same application, VPM and SITUVIS can be considered orthogonal systems: on the one hand, SITUVIS is very effective in 
showing the state/s of the smart space in a given situation, on the other hand, VPM is particularly suited to show the correlations between actions in the meanwhile than an activity is performed as well as the precedence relations between the activities constituting a complete daily habit of users acting into a smart environment.

The main contribution of this paper, presented in this section, is a comparison, conducted as a user study, between the two solutions, showing commonalities and differences. The user evaluation study for both the systems has been based on a questionnaire, which is is available at the link https://goo.gl/forms/yg4fBkkDMF74I8c92 ${ }^{2}$

\subsection{Test structure}

The user evaluation has been conducted during late October and early November 2017, and it involved a group of 14 testers: the questionnaire has been fed to them in three rounds, 4 people in the first one, 5 each in the following two ones. The group of users is composed by people heterogeneously distributed in terms of age, expertise level and gender. Each tester was required to indicate these information at the beginning of the questionnaire. All testers performed the test in the same room to ensure the same environmental conditions to everyone. During the first part of the experiment, a brief non-technical description of the two visualization systems was provided, to explain their functionalities and representations. Before the beginning of each test, a question\&answer session was conducted.

The test is structured to investigate the graphical expressiveness of the two representations offered by VPM and SITUVIS. It is divided in two specular parts, so that for each system the same features have been investigated. Each part is structured as it follows:

- the first two questions propose an unlabeled example where the user is asked to indicate which is the most likely label between the provided ones;

- the following two questions conversely adopt the inverse schema: the users are requested to indicate which one of the proposed representations, in their opinion, is the one corresponding to a given activity label;

- in the third section, some questions are posed about the correlation between activities and the clarity of the system state according to the model proposed;

- in the fourth section, an interpretation of two proposed outputs is shown, where only one of them is correct. The tester can indicate his/her confidence level about each graph interpretation;

- in the last section, some questions are proposed to obtain an expressiveness evaluation of both systems (i.e., connections and relevance of features).

\subsection{Comparison results}

In this section, the results obtained be performing previously described tests are presented and commented. They

2. A printed version is available at https://drive.google.com/open?id= 11 gccgTsfbvCfUM5c-0e3yes6SX-e7_Tf
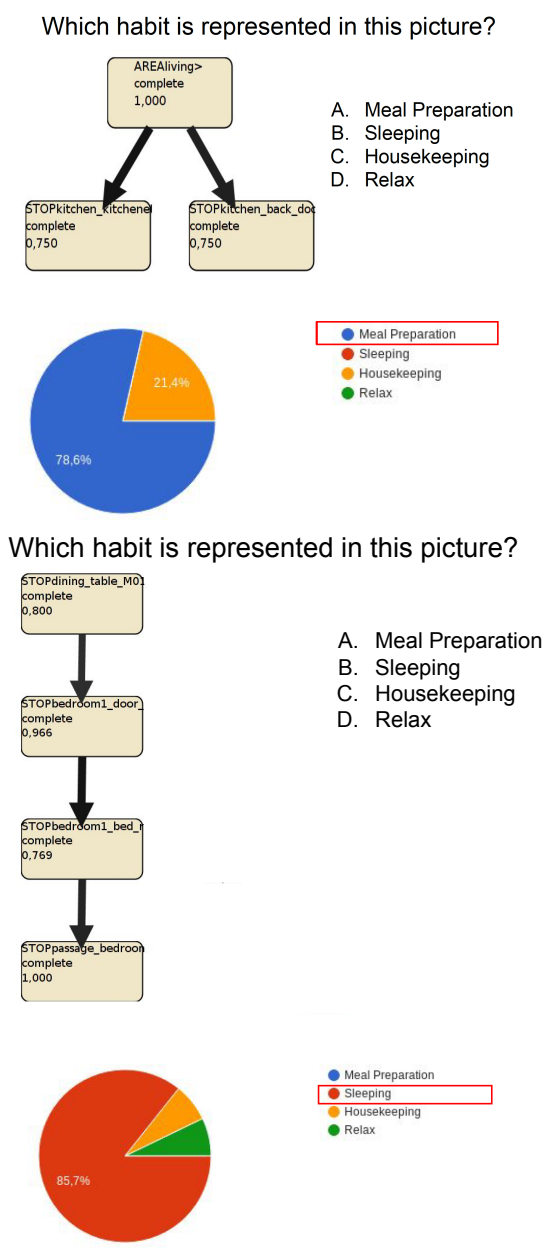

Figure 1: Users test result about VPM models clarity: the results show how the model produced are easily readable, since they are correctly interpreted by the $78 \%$ of the users in the first test and by the $85.7 \%$ in the second one.

are graphically shown in the Figures 113 (see further). The structure of each figure presents the question text and the graph of the distribution of the results, notably some figures can contain more questions. We start with VPM, cf. Figures 177, which is our contribution.

The questions in Figure 1 aim at investigating clarity: an habit is proposed and the tester is required to indicate which is the correct habit between the four proposed: results show how the model produced are easily readable, since they are correctly interpreted by the $78 \%$ of the users in the first test and by the $85.7 \%$ in the second one. Also the two following questions proposed in Figure 2 have the same purpose, but are structured in an inverse way: an habit is declared, the user have to indicate which model fits that habit. The results show how the models produced are easily readable, since they are correctly interpreted by the $79.6 \%$ of the users in the first test and by the $100 \%$ in the second one.

Figure 3 shows the results obtained about users confidence in interpreting the model and in understanding the 
Which of the following pictures represents the habit "Eating"?
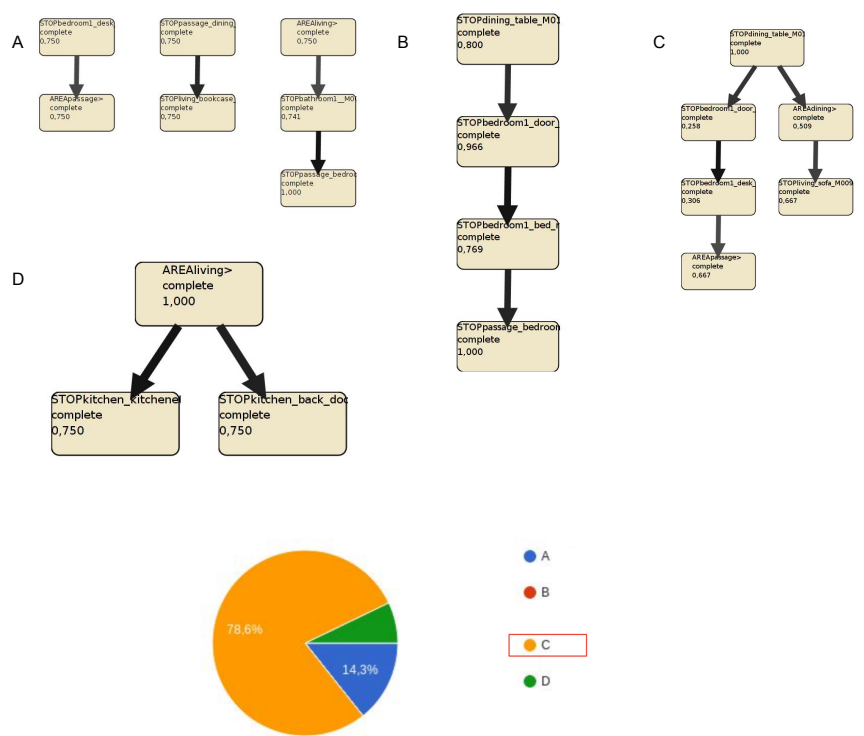

Which of the following pictures represents the habit "Sleeping"?
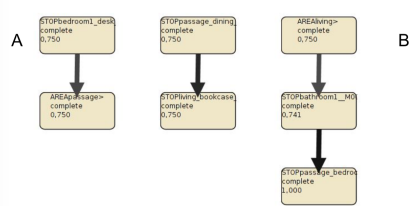

D

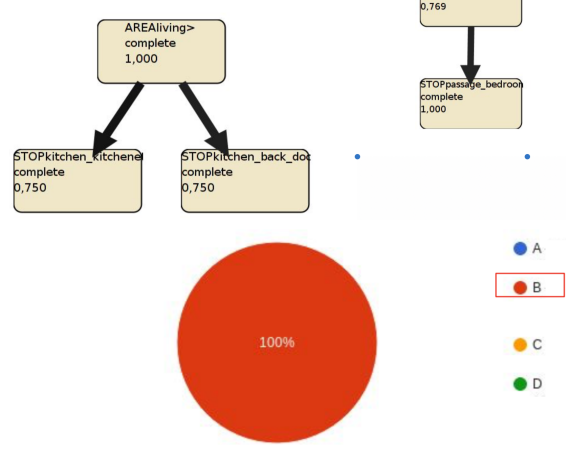

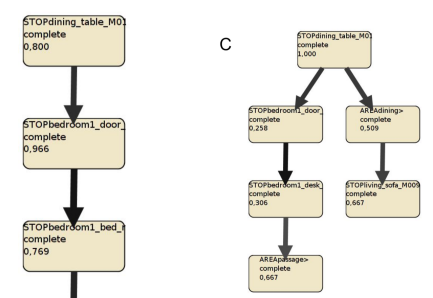

Figure 2: Other two users test result about this system models clarity: the results show how the model produced are easily readable, since they are correctly interpreted by the $79.6 \%$ of the users in the first test and by the $100 \%$ in the second one.

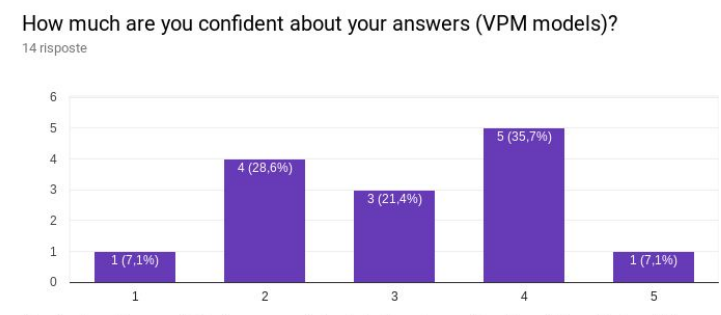

Analyzing the models, how much is intuitive to understand the state of the system?

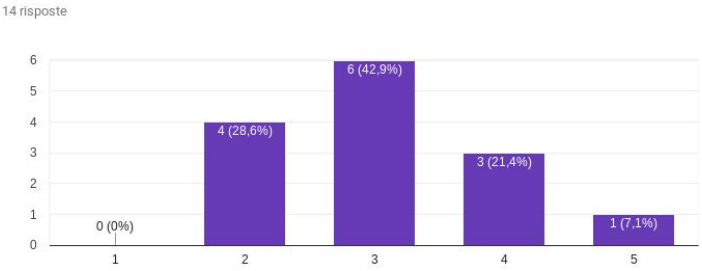

Figure 3: Graphs about users confidence in interpreting the model and in understanding the system state: as expected, the first one is resulted to be quite high, with the $72.7 \%$ of them declaring medium or high $(>3)$ confidence in their answers and the second one, about the state, is less performing, with the $71.5 \%$ reputing medium or low $(<3)$ the intuitiveness about this aspect

system state: as expected, the first one is resulted to be quite high, with the $72.7 \%$ of them declaring medium or high $(>3)$ confidence in their answers and the second one, about the state, is less performing, with the $71.5 \%$ reputing medium or low $(<3)$ the intuitiveness about this aspect. The aspects shown by Figure 4 touch the correlation between habits. The results show how the user gets it: the $42.9 \%$ see the models as unrelated but the $85.8 \%$ thinks the models can represent in an adequate model the correlation (rate $>3$ ).

An interesting aspect to investigate is how the fuzzy mining technique applied to smart spaces is interpreted by users. An hypothetical model and an interpretation, not necessarily completely fitting to it, is proposed to the user. S/he has to indicate an agreement level wrt. the proposed sentence. The results obtained are shown in Figure 5 , the users responded very well in case of a description completely fitting the graph (Figure ??, all the participants give an agreement medium or high $(>3)$, and the $78.6 \%$ expressed the maximum agreement level). However the interpretation has been less precise in the case of a not completely correct description (results are symmetrically distributed, cf. Figure ??.)

The following question covers how much in the model appears clear the correlation between different actions in an habit. The results, shown in Figure 6 are good, with the $71.4 \%$ correct answers for the first question and the 64.3\% for the second one. This demonstrates how the technique is feasible to model the consequentiality between the different actions in an habit.

The last part of the user evaluation about VPM is focused on investigating how much the fuzzy model are clear on the importance of a given activity in an habit, and the confidence level of the testers. Figure 7 contains the results: 


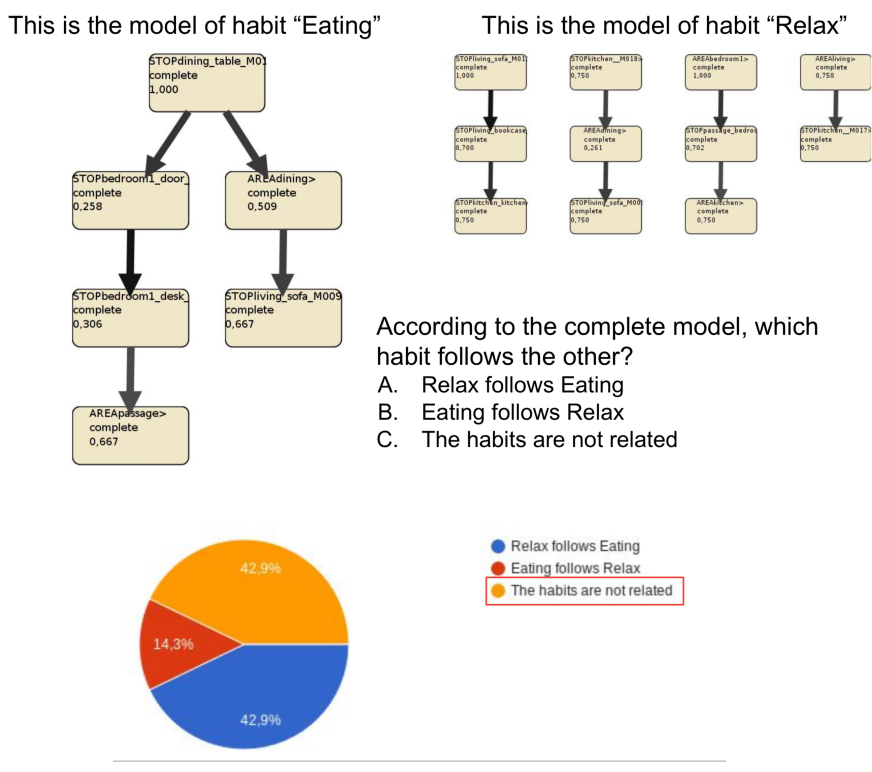

Analyzing the models, how much is intuitive to understand the evolution of the habits?

14 risposte

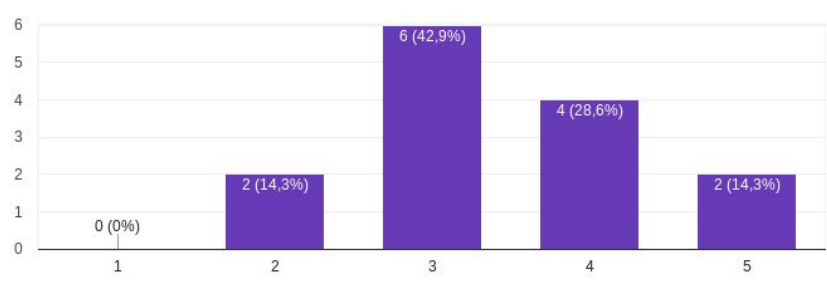

Figure 4: User test about the correlation between habits: the results show how the user gets the correlations between different habits: the $42.9 \%$ see the models as unrelated but the $85.8 \%$ thinks the models can represent in an adequate model the correlation (rate $>3$ )

the $57.1 \%$ of the participants has given the correct answer and also the confidence level is very high (the $85.8 \%$ has given a medium or high rate, $>3$ ). This demonstrates that this aspect is well managed by VPM, and VPM also gives some certainty to final users.

After VPM analysis, the test continues in evaluating SITUVIS, cf. Figures $8-13$ As previously explained, the test structure is specular to the VPM part. So, in the first questions, SITUVIS graph clarity is investigated: the representation of a situation is proposed and the tester is required to indicate which is the correct one between the four proposed. Figure 8 results show how the representations are very readable, since they are correctly interpreted by the
How much do you think the following description fits with the model in figure? "The user performs an action near the dining table. Then or moves in the dining room, or in the bedroom: in first case, he/she performs one or more actions in the room, ending on the living room sofa; In the second one he/she performs one or more actions near the door, then performs one or more actions on the desk, ending with an action in the passage"
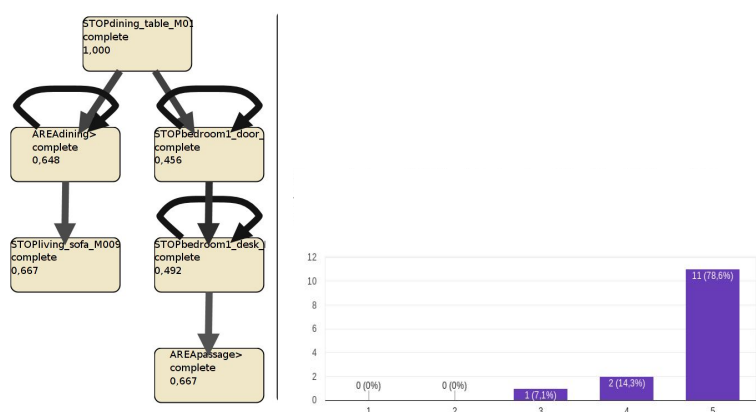

How much do you think the following description fits with the model in figure? "The user performs an action on the dining table, then performs an unique action near the first bedroom door and a single action on the bed."
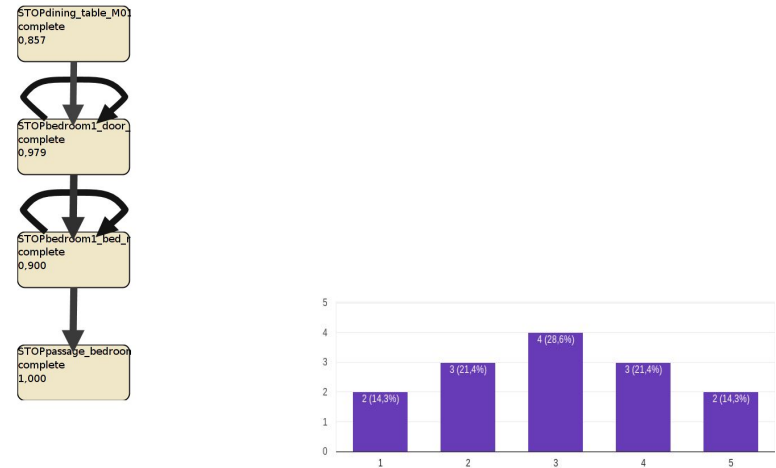

Figure 5: User tests results about the interpretation of fuzzy graph applied to smart spaces: the user responded very well in case of a description completely fitting the graph (all the participants give an agree medium high $(>3)$ and the $78.6 \%$ has expressed the maximum agreement level). However the interpretation has been less precise in case of a not completely correct description (results are symmetrically distributed.)

92.9\% in both cases. Following the VPM test structure, also the two next questions proposed in Figure 9 have the same purpose, but are structured in an inverse way: a situation is declared, the user has to indicate which model fits better. Results confirm its high readability, since the correct answer has been given by the $92.9 \%$ in one case and by the $71.4 \%$ in the other one.

After that, the correlation between situations has been checked. Two situations are proposed, and the user is required to indicate which is the correlation between them, if any. Figure 10 contains results about that: as expected, in the user tests on correlation between activities $71.4 \%$ of the testers has not been able to get the correlation between 
According to the complete model, which is the action most correlated to Area Living?
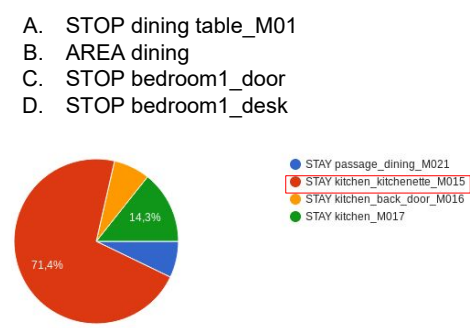

- StaYktchen-M017

According to the complete model, which is the action less correlated to Area Living?
A. STOP dining table_M01
B. AREA dining
C. STOP bedroom1 door
D. STOP bedroom1_desk
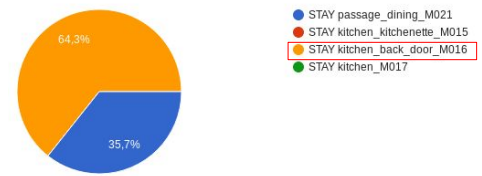

Figure 6: User evaluation that reports the how much the model makes clear the correlation between different actions in an habit. The results are good, with the $71.4 \%$ fore the first question and the $64.3 \%$ for the second of correct answers.

two activities. From the one hand, SITUVIS is particularly good in visualizing system state of the single situation, on the other hand it results difficult to utilize for getting interactions/relationships among situations. This fact is also confirmed by the results in Figure 11, about the expressiveness level of SITUVIS about both the activity state and the activity evolutions: the results confirm hypothesis that the graph is very effective on the state $(78.5 \%$ rated a value medium or high $>3$ ) but does not give many information on situations correlations $(57.2 \%$ rated low or medium, $<3$ ).

The following question is about general activity interpretation: a model and a description, not necessarily fitting, are proposed to the tester and s/he should express an agreement level. Quite precise scores, shown in Figure 12, have been achieved: the first question (Figure 12a) contains a wrong activity description, and the participants have been able to recognize it, since the $78.6 \%$ has expressed an agreement level low or medium $(<3)$. Also in the other question (Figure $12 \mathrm{~b}$ ) in which the description relative to the model proposed is correct, has been well recognized since the $92.8 \%$ of the testers expressed an agreement medium or high with a peak of $71.4 \%$ on value 5 .

The last section of the test is centered on understanding user confidence in interpreting SITUVIS output and his expressiveness. Graphs in Figure 13 show the confidence level obtained by the testers on SITUVIS and its expressiveness level: for the first question, the $92.9 \%$ of the participants has given a medium or high grade $(>3)$. Also the expressiveness
Consider the model in the figure, which is the action less representative of the habit?

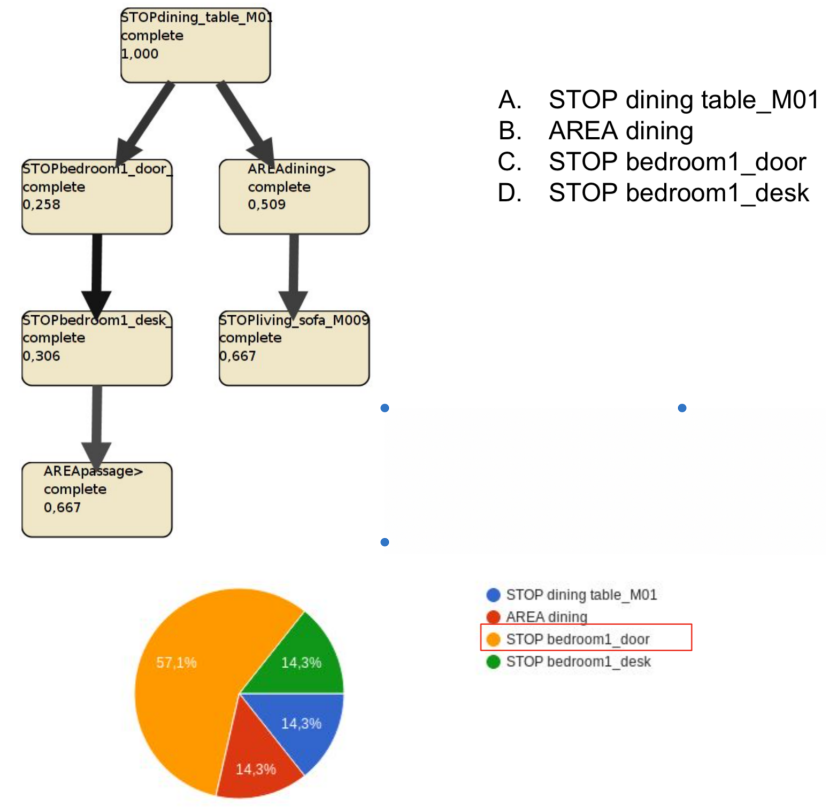

How much are you confident about your answers (VPM models)? 14 risposte

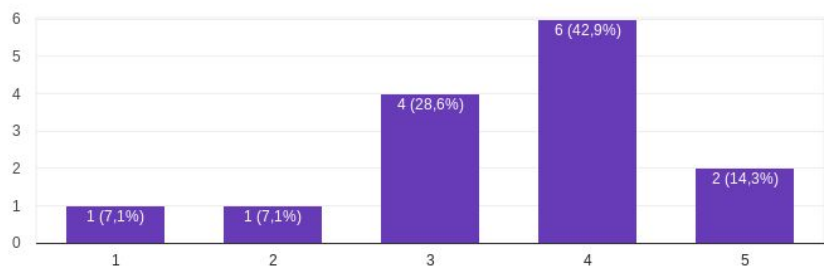

Figure 7: User evaluation that reports how much the fuzzy model makes clear the importance of a given activity in an habit: the $57.1 \%$ of the participants has given the correct answer and also the confidence level is very high (the $85.8 \%$ has given an medium or high rate, $>3$ )

level has reached similar results, since the $85.8 \%$ of the participants considered it medium or high. So SITUVIS system results to be an expressive tool, that gives user the certainty of what he is interpreting.

\section{Conclusions}

In this paper, we have presented the results of an user evaluation aimed at investigating which representation is preferable, for a service of visual analytics in smart spaces, 
Which activity is represented in this picture?

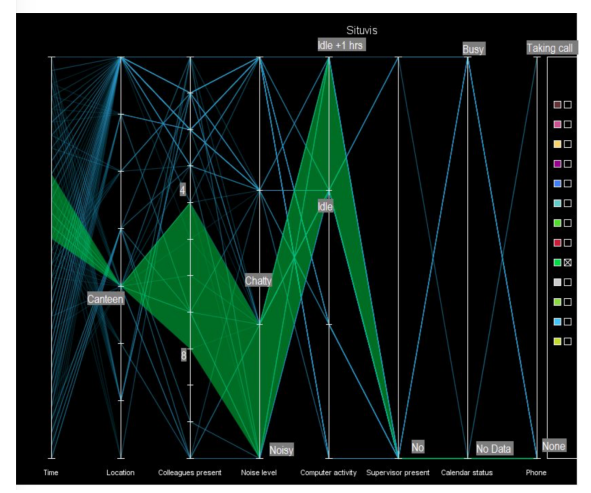
A. Paper Writing
B. Phone Call
C. Lunch
D. Playing Football

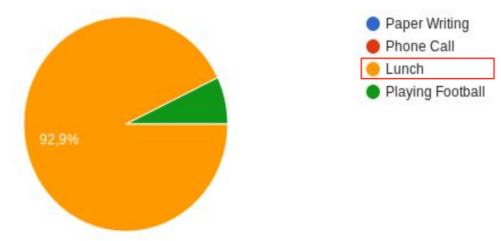

Which activity is represented in this picture?

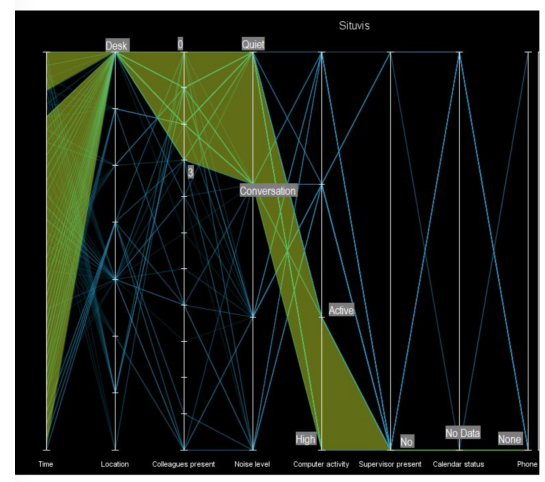
A. Paper Writing
B. Phone Call
C. Lunch
D. Playing Football

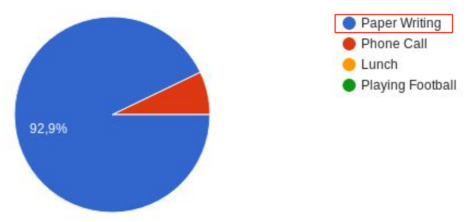

Figure 8: User test result about SITUVIS graph clarity: the results show how the model produced are very readable, since they are correctly interpreted by the $92.9 \%$ in both cases

between activity-based and situation-based. We have based the evaluation on two available systems, namely VPM and SITUVIS. Ultimately, the two systems, for some aspects have similar applications. Both of them are used for representing events inside a smart space, and are designed for providing the user a tool for performing deep space analysis. However, they have some peculiarities that make them orthogonal: in fact, if SITUVIS is very effective in giving a "screenshot" of the system state for a situation, VPM results to be more effective in representing correlations between the

Which of the following pictures represents the action "Phone Call"?
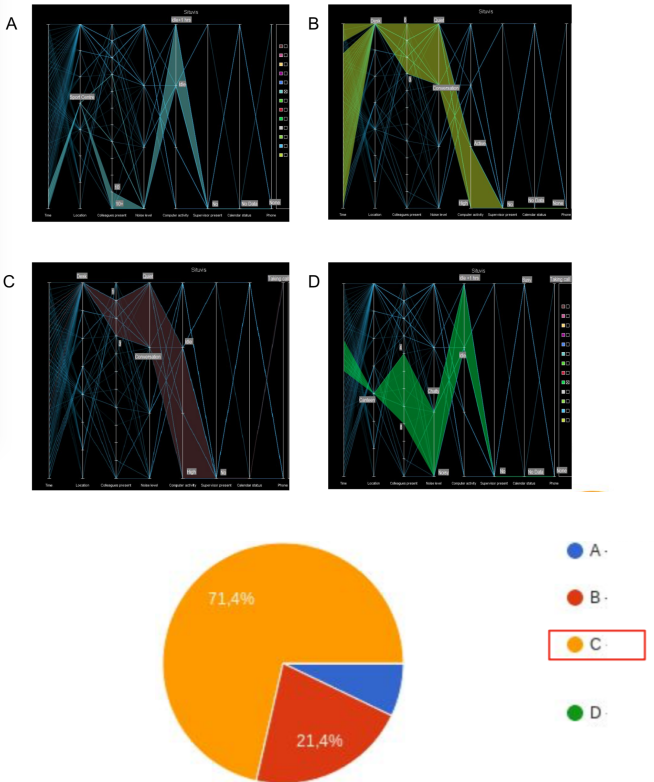

Which of the following pictures represents the action "Lunch"?
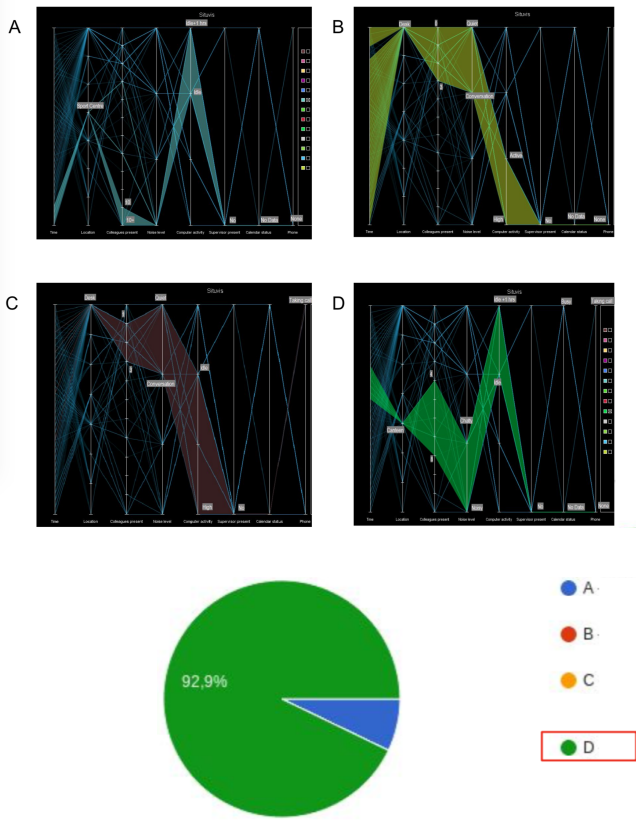

Figure 9: Other user test about SITUVIS graph clarity: the results confirm its high readability, since the correct answer has been given by the $92.9 \%$ in one case and by the $71.4 \%$ in the other one. 

This is the representation of the activity
"Paper Writing"

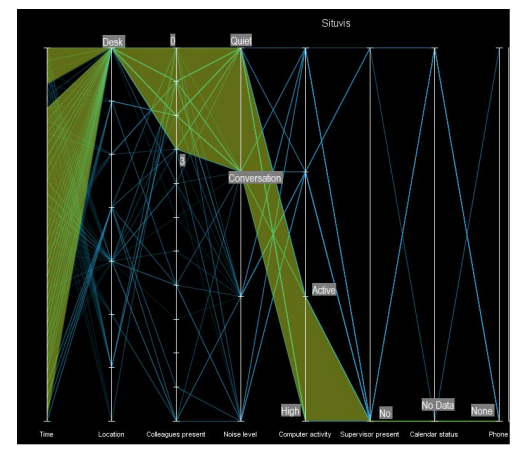

According to the complete model, which habit follows the other?

$$
\begin{aligned}
& \text { A. Paper Writing follows Lunch } \\
& \text { B. Lunch follows Paper Writing } \\
& \text { C. The habits are not related }
\end{aligned}
$$

This is the representation of the activity "Paper Writing"
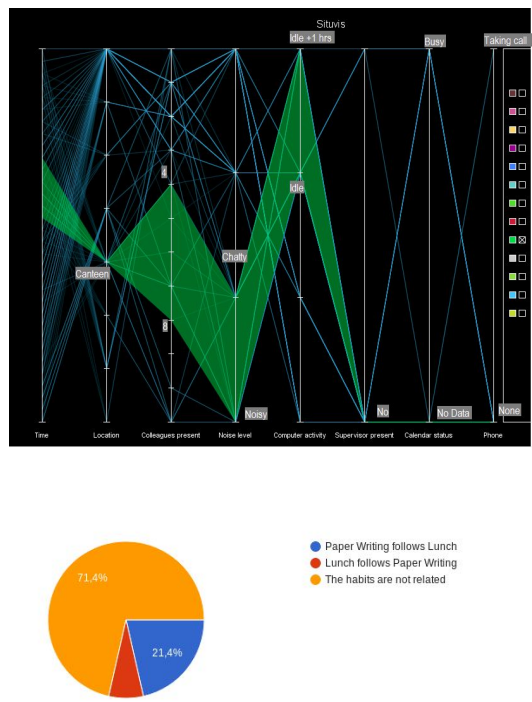

Figure 10: As expected, the user tests on correlation between activities has returned the a result in which the $71.4 \%$ of the testers has not been able to get the correlation between two activities.

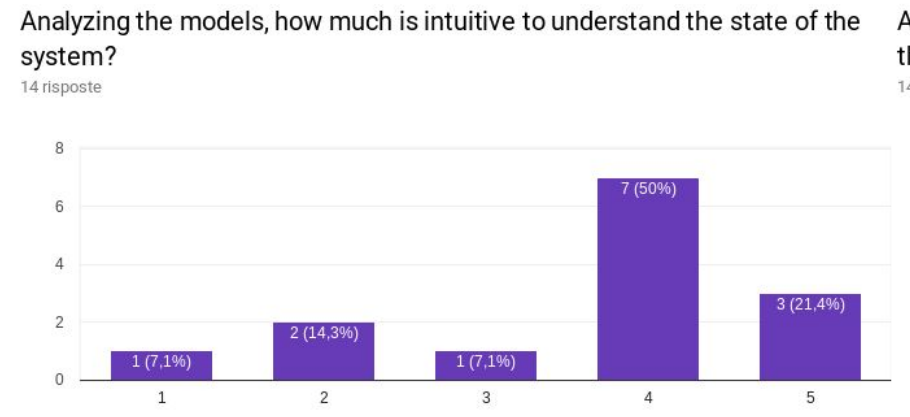

(a)
Analyzing the models, how much is intuitive to understand the evolution of
the activities?
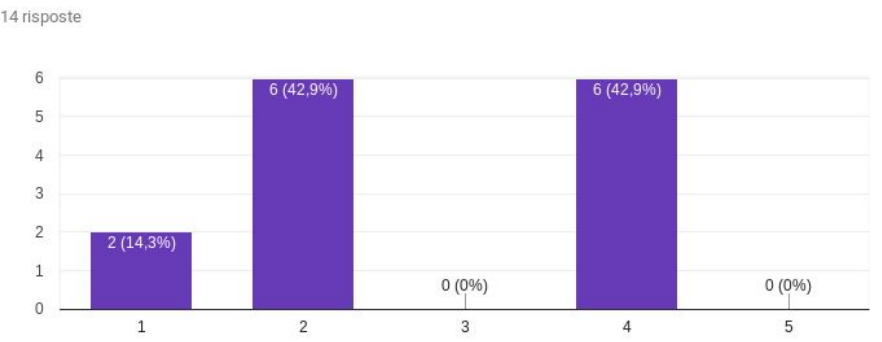

(b)

Figure 11: Graphs reporting the tests results about the expressiveness level of SiTUVISabout both the activity state and the activities evolution: the results confirm hypothesis that the method is very effective on the state ( $78.5 \%$ rated a value medium or high $>3$ ) but does not give many information on situations correlations $(57.2 \%$ rated low or medium, $<3)$

components of an habit and their evolutions. Notably, VPM is not under performing in terms of clarity, expressiveness, and user confidence wrt. SITUVIS.

\section{References}

[1] A. K. Clear, T. Holland, S. Dobson, A. Quigley, R. Shannon, and P. Nixon, "Situvis: A sensor data analysis and abstraction tool for pervasive computing systems", Pervasive and Mobile Computing, vol. 6 , no. 5, pp. 575-589, 2010.

[2] S. W. Loke, "Logic programming for context-aware pervasive computing: Language support, characterizing situations, and integration with the web", in Proc. 2004 IEEE/WIC/ACM International Conference on Web Intelligence, 2004, pp. 44-50.

[3] S. Loke, "Incremental awareness and compositionality: A design philosophy for context-aware pervasive systems", Pervasive and Mobile Computing, vol. 6, no. 2, pp. 239-253, 2010.
[4] D. Riboni and C. Bettini, "Context-aware activity recognition through a combination of ontological and statistical reasoning", Ubiquitous Intelligence and Computing, pp. 39-53, 2009.

[5] R. Helaoui, D. Riboni, and H. Stuckenschmidt, "A probabilistic ontological framework for the recognition of multilevel human activities", in Proc. 2013 ACM International Joint Conference on Pervasive and Ubiquitous Computing, 2013, pp. 345-354.

[6] D. Riboni, T. Sztyler, G. Civitarese, and H. Stuckenschmidt, "Unsupervised recognition of interleaved activities of daily living through ontological and probabilistic reasoning", in Proc. 2016 ACM International Joint Conference on Pervasive and Ubiquitous Computing.

[7] J. C. Augusto and C. D. Nugent, "The use of temporal reasoning and management of complex events in smart homes", Proc. ECAI 2004.

[8] A. Aztiria, J. C. Augusto, R. Basagoiti, A. Izaguirre, and D. J. Cook, "Discovering frequent user-environment interactions in intelligent environments", Personal and Ubiquitous Computing, vol. 16, no. 1, pp. 91-103, 2012. 
How much do you think the following description fits with the model in figure?

"This activity is performed during the evening in the sport center by ten or more people. This activity is quite silent, and requires strong pc use. This activity is not performed with the supervisor, doesn't represent a calendar appointment and doesn't requires the usage of the phone"
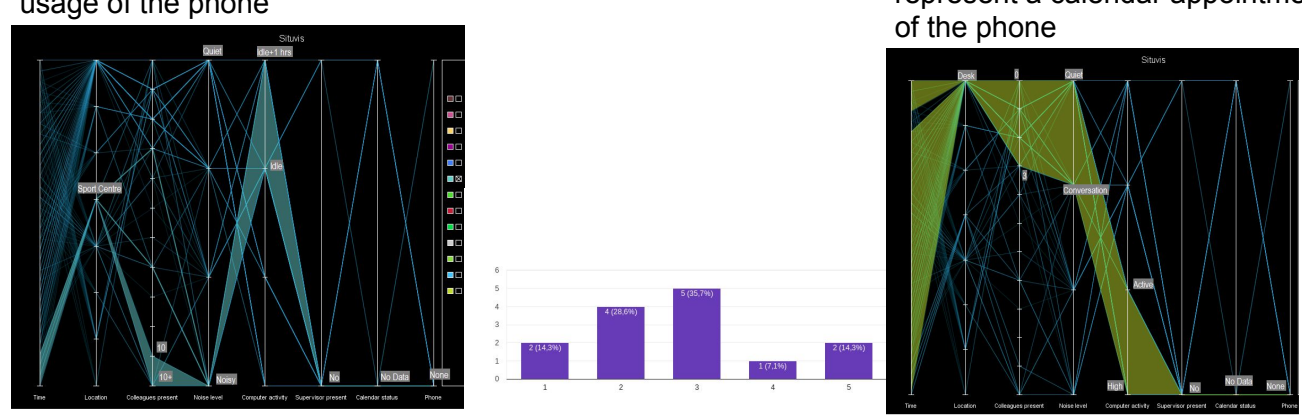

(a)
How much do you think the following description fits with the model in figure?

"This activity is performed the most of the time on the office desk. When the activity is performed, in the room there are few people (less than 3). This activity is quite silent, and requires strong pc use. This activity is not performed with the supervisor, doesn't represent a calendar appointment and doesn't requires the usage

(b)

Figure 12: Test results about general activity interpretation. Scores achieved are quite precise. The participants have been able to recognize a wrong activity description, since the $78.6 \%$ has expressed an agreement level low or medium $(<3)$. Also the other description relative to the graph and correct, has been well recognized since the $92.8 \%$ of the testers expressed and agreement medium or high with a peak of $71.4 \%$ on value 5

How much are you confident about your answers (SITUVIS)?

14 risposte

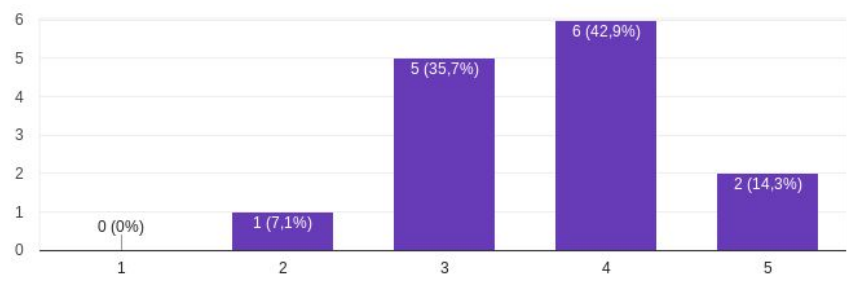

(a)
How much do you think SITUVIS system is expressive?

14 risposte

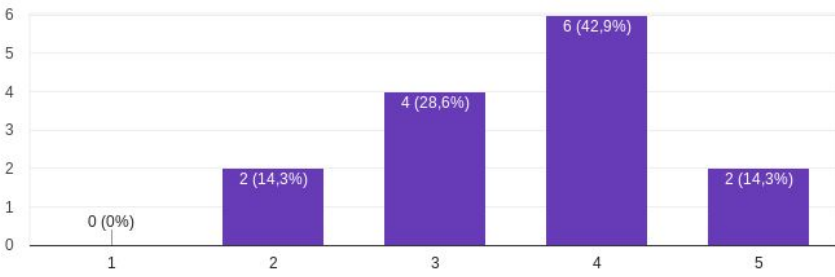

(b)

Figure 13: Graphs showing the confidence level obtained by the testers on SITUVISvisualization tool and its expressiveness level: for the first question, the $92.9 \%$ of the participants has given a medium or high grade ( $>3$ ). Also the expressiveness level has reached similar results, since the $85.8 \%$ of the participants considered it medium or high.

[9] F. Leotta, M. Mecella, and J. Mendling, "Applying process mining to smart spaces: Perspectives and research challenges", in CAiSE 2015 Workshops, Springer, 2015, pp. 298-304.

[10] M. Dumas, M. La Rosa, J. Mendling, H. A. Reijers et al., Fundamentals of business process management, Springer, 2013.

[11] W. M. van der Aalst, Process mining. Springer, 2016.

[12] B. Gottfried, H. Guesgen, and S. Hbner, "Spatiotemporal reasoning for smart homes", in Designing Smart Homes, LNAI 4008, J. Augusto and C. Nugent, Eds. 2006, pp. 16-34.

[13] A. Aztiria, A. Izaguirre, R. Basagoiti, J. C. Augusto, and D. J. Cook, "Discovering frequent sets of actions in intelligent environments", in Intelligent Environments, 2009, pp. 153-160.

[14] A. Aztiria, A. Izaguirre, R. Basagoiti, J. C. Augusto, and D. Cook, "Automatic modeling of frequent user behaviours in intelligent environments" in Intelligent Environments, 2010, pp. 7-12.

[15] N. Tax, E. Alasgarov, N. Sidorova, and R. Haakma, "On generation of time-based label refinements", arXiv preprint arXiv:1609.03333, 2016
[16] N. Tax, N. Sidorova, R. Haakma, and W. M. van der Aalst, "Event abstraction for process mining using supervised learning techniques", arXiv preprint arXiv:1606.07283, 2016.

[17] C. W. Günther and W. M. Van Der Aalst, "Fuzzy mining-adaptive process simplification based on multi-perspective metrics", in BPM 2007, Springer, 2007, pp. 328-343.

[18] M. Dimaggio, F. Leotta, M. Mecella, and D. Sora, "Process-based habit mining: Experiments and techniques", in Ubiquitous Intelligence \& Computing (UIC), 2016 Intl IEEE Conference.

[19] F. Leotta, M. Mecella, D. Sora, and G. Spinelli, "Pipelining user trajectory analysis and visual process maps for habit mining", in Ubiquitous Intelligence \& Computing, 2017 Intl IEEE Conference.

[20] J. Ye, S. Dobson, and S. McKeever, "Situation identification techniques in pervasive computing: A review", Pervasive and mobile computing, vol. 8, no. 1, pp. 36-66, 2012. 